\title{
Alimentary Limb Ischemia During Roux-en-Y Gastric Bypass and its Management
}

\author{
Tarun Mittal $^{1}$ (D) $\cdot$ Ashish Dey ${ }^{1} \cdot$ Abhilash Nali $^{1} \cdot$ Sheikh Mohammad Taha Mustafa ${ }^{1} \cdot \operatorname{Vinod~K}$ Malik $^{1}$
}

Published online: 13 March 2018

(C) Springer Science+Business Media, LLC, part of Springer Nature 2018

\begin{abstract}
Introduction Ischemia of the tip of the alimentary limb involving the gastrojejunostomy (GJ) is an unusual complication during Roux-en-Y gastric bypass (RYGB). Revision of the GJ may be needed to manage this complication.

Objectives We present a case of inadvertent perforation of the jejunum by a gastric calibration tube, which was recognized on the table and appropriate measures are then taken. Repair of this enterotomy however then led to ischemia of the tip of alimentary limb extending to a portion of the GJ anastomosis. We present its subsequent management.

Methods Our patient is a morbidly obese female patient with a BMI value of $44.6 \mathrm{~kg} / \mathrm{msq}$ undergoing RYGB. We performed an antecolic, antegastric linear-stapled gastrojejunostomy of $2.5 \mathrm{~cm}$. After hand-sewn closure of the common enterotomy, we tried passing a 38-F Bougie through the anastomosis into the Roux limb. During this maneuver, the Bougie inadvertently perforated the Roux limb at the mesenteric border. Following the repair of this jejunal rent, the distal part of the Roux limb became dusky and involved a portion of the gastrojejunal anastomosis. The gastrojejunostomy was therefore completely revised. Intraoperatively, methylene blue dye test was done which showed no leak.

Results Post-operative gastrografin study revealed no leak and liquids were started on POD1.

Conclusion Inadvertent injury by a Bougie is a rare but known complication in laparoscopic RYGB. Intraoperative recognition of the complication is essential to prompt the necessary repair. Revision of the gastrojejunostomy is necessary if the Roux limb or the anastomosis itself is ischemic and can be accomplished with good results.
\end{abstract}

Keywords Alimentary limb ischemia $\cdot$ Roux-en-Y gastric bypass $\cdot$ RYGB $\cdot$ Bougie $\cdot$ Management

All authors are in agreement with changes in author order/author name, change in spelling, and degrees of authors.

Electronic supplementary material The online version of this article (https://doi.org/10.1007/s11695-018-3197-9) contains supplementary material, which is available to authorized users.

Tarun Mittal

tarunmittalmas@gmail.com

Ashish Dey

ashishdey_78@rediffmail.com

Abhilash Nali

abhilashchintu12@gmail.com

Sheikh Mohammad Taha Mustafa

sheikhtaha23@gmail.com

Vinod K. Malik

vinod.k.malik@gmail.com

1 Department of General and Laparoscopic Surgery, Sir Ganga Ram Hospital, New Delhi 110060, India

\section{Compliance with Ethical Standards}

Conflict of Interest The authors declare that they have no conflict of interest.

Informed Consent Appropriate informed consent was taken.

Ethical Approval This article does not contain any studies with human participants or animals performed by any of the authors. 